\title{
Medical-biological significance of specific CIC and good perspectives of immune complex disintegration using ultrasonic in laboratory diagnostics of HIV-infection*
}

\author{
V.L.Pastushenkov ${ }^{1}$, Yu. A.Mitin', A.V.Semenov, ${ }^{3,4}$, \\ D. E. Valutite ${ }^{3}$, S. Yu. Dudnikov', M. M. Gabaeva ${ }^{5}$ \\ ${ }^{1}$ Sevastopol State University, \\ 33, Universitetskaya ul., Sevastopol, 299053, Russian Federation \\ 2 S. M. Kirov Military Medical Academy, \\ 6, ul. Academika Lebedeva, St. Petersburg, 194044, Russian Federation \\ ${ }^{3}$ St. Petersburg Pasteur Institute, \\ 14, ul. Mira, St. Petersburg, 197101, Russian Federation \\ ${ }^{4}$ North-Western State Medical University named after I. I. Mechnikov, \\ 41, Kirochnaya ul., St. Petersburg, 191015, Russian Federation \\ ${ }^{5}$ Kabardino-Balkarian State University named after H. M. Berbekov, \\ 173, ul. Chernyshevskogo, Nalchik, Kabardino-Balkarian Republic, 360004, Russian Federation
}

For citation: Pastushenkov V.L., Mitin Yu. A., Semenov A. V., Valutite D. E., Dudnikov S. Yu., Gabaeva M. M. Medical-biological significance of specific CIC and good perspectives of immune complex disintegration using ultrasonic in laboratory diagnostics of HIV-infection. Vestnik of Saint Petersburg University. Medicine, 2020, vol. 15, issue 4, pp. 239-245. https://doi.org/10.21638/spbu11.2020.402

The article contains data, characterizing results variations of specific laboratory HIV-infection diagnostics after ultrasonic exposure directed at biological objects of blood serum. Detailed consideration of biophysical and medical aspects of tissues response on ultrasonic exposure, including immune complexes dissociation, accompanied by antibodies and antigens appearance, previously being in bound state and inaccessible for revealing by laboratory methods was carried out. On the example of HIV-infection good perspectives of ultrasonic application for immune complexes disintegration in laboratory diagnostics is demonstrated.

Keywords: ultrasound, circulating immune complexes, dissociation, laboratory diagnostics, HIV-infection, antibodies, antigens.

* The research was performed with financial support of Federal State Autonomous Educational Institution of High Education "Sevastopol State University" within initiative scientific project no. 59/06-31.

(C) St. Petersburg State University, 2020 
One of the most actual problems in medicine is diagnostics and treatment of HIVinfection [1]. Studding of relatively new for human disease and one of the most complex among the known infections relates to higher-priority directions of the science. It is due to, firstly, that HIV control is a vitally important task of healthcare; and, secondly, that approaches, developed for HIV control, would be high-demanded in therapy and in prevention of the other diseases [2].

One of the problems, arising at primary laboratory examination of patient at earlier stages of HIV-infection, is a possibility to obtain false-negative results at the period of disease when infecting of patient already happened, but the results of examination on antibodies to HIV are still negative [3; 4]. This phenomenon may take place in the period of the so called "serological window", arising at early stages of infection, when infecting happened relatively recently, antibodies level is low and formed antibodies became associated with HIV antigens and formed immune complexes. In formation of such complexes antibodies to HIV become inaccessible for revealing. The same processes may be observed at terminal stage of HIV-infection, when antibodies level is lower or equal to antigens level, that leads to bonding of all or majority of free antibodies to immune complexes, complicating their detection by means of enzyme-linked immunosorbent assay [5].

Formation of immune complexes in course of different antigens and antibodies interaction presents important immunological phenomenon, considerably determining the course of pathological process at different diseases. This process, for example, in case of viral infections, is one of the mechanisms of virus activity biological neutralization [6].

Bonded HIV antigens with viral antibodies lead to CIC formation. Thous complexes continuously present in blood serum of patients with HIV-infection. On the basis of last year's scientific investigations data, it is possible to point out the following. Continuous presence of HIV antigens in infected persons is accompanied by continuous formation of anti-viruses antibodies. Performed by a group of authors determination of CIC in HIVinfected patients enabled to estimate amount and qualitative composition of immune aggregates [7]. Spectroturbidimetrical analysis of CIC precipitation with polyethylene glycol did not reveal statistically significant differences in mean particle diameter of immune aggregates in HIV-infected patients with different level of viral load (VL). Meanwhile, determination of immunoglobulin levels within CIC demonstrated, that in all patients presented different size complexes mainly containing IgG by IgM. Therewith, in patients with high VL level, concentration of such CIC was considerably higher as compared with respective indices in patients with suppressed virus replication. The authors supposed that on background of performed antiretroviral therapy, reduction of HIV antigens amount leaded to reduction of antibodies production and to respectively expressed reduction of antigen-antibodies complexes concentration. At the same time, they noted, that in patients with large amount of HIV in blood, main part of complexes was presented by lowmolecular IgG-containing structures. Thus, this study confirmed diagnostic significance of CIC at HIV-infection and dynamics of their content, associated with different stages infection and also with antiretroviral therapy influence.

Another investigation contained comparative analysis of "free" antibodies and "bounded" with proteins virus frequency occurrence in blood serum circulated immune complexes of HIV-infected patients at early period of the disease [8]. In accordance with WHO recommendations on immunoblot results interpretation, the authors of the given 
investigation revealed 20 probes with ambiguous result and 26 probes with positive results of immunoblot. In spite of the fact that blood serum were obtained at different periods of dispensary observation of HIV-infected patients, nonetheless, in the first group antibodies to gp 160 , p55, p25 were mainly revealed, and in the second group additionally to gp 120 , p68 and p52. At that, in the group with positive immunoblot results, frequency of antibodies to gp160, gp120 and p25 occurrence in blood serum constituted $100 \%$. Titers of "free" antibodies to HIV in patients of the first group varied, according to enzyme-linked immunosorbent assay, from 1:4 to 1:128, and in patients in the second group they were in the range from 1:256 to 1:4096, accordingly. These investigations confirmed information that dynamics of infecting process is accompanied by titer growth of "free" antibodies to HIV already at early stages of the disease.

\section{Purpose of investigation}

Experimental studying of ultrasonic exposure on biological objects of blood serum in HIV-infected patients.

Optimization of early laboratory diagnostics of HIV-infection remains actual task for practical healthcare. The most important clause of the Russian Federation laboratory service governing documents with respect to HIV-infection is the following: "Standard laboratory HIV-infection diagnostics method is determination antibodies/antigens to HIV by means of enzyme-linked immunosorbent assay. For results confirming with relation to HIV, confirming tests are used (immune, linear blot)". Thus, screening strategy is provided by means of immunofluorescence assay. As it was pointed out earlier, one of the problems, arising at primary patients diagnostics at early stages of HIV-infection, is a possibility to obtain false-negative results in the period of the so called "serological window". Specific antibodies bounded with HIV antigens, forming circulating immune complexes, within which composition antibodies to HIV become unacceptable for detection by laboratory diagnostics methods. The same processes may be distinctive also at terminal stage of HIVinfection, when antibodies level is lower or equal to antigens level, leading to bonding of all or majority of free antibodies to immune complexes, complicating their detection by means of immunofluorescence assay [5].

Is it possible to perform such an impact on CIC in blood serum of HIV-infected patients to cause its disintegration and obtain from its composition free antibodies, provided that they would remain their biological activity and ability to participate in immure reactions? We suppose, that methodologically the work should be directed at looking for impact mostly sparing biological objects physiologically. Among the most prospective directions, meeting these conditions, is investigation ultrasonic exposure on CIC of blood serum in HIV-infected patients [9].

In course ultrasonic exposure on liquid biological medias takes place the most important effect - cavitation, defined as formation of single or multiple gas bubbles (cavities) in liquid volume. Processes of cavitation, arising in liquid biological media under ultrasonic exposure, lead to chemical processes enhancement in it, formation of different radicals, and, as a result, to modification of macromolecules state, presented in the given biological media. Originating chemical and thermo-dynamical processes are implemented in the form of macromolecules dissociation with ability of their subsequent recombination, accompanied by chemoluminescence phenomena. Results of 
such exposure may become macromolecules dissociation, i. e., disintegration of immune complexes on their constituents - antigens and antibodies. Appearance of free antigens and antibodies, previously being in bounded state, may be used for laboratory diagnostics. Such antigens and antibodies may be already revealed using methods of immune analysis - enzyme-linked immunosorbent assay (ELISA), immunoblotting, immunofluorescence assay [10].

\section{Material and methods}

Probes of blood serum taken from 28 HIV-infected persons being under dispensary dynamics observation in State public healthcare institution of Leningrad region (Saint-Petersburg) "Tsentr SPID", were included in this investigation. For each blood serum sample previously primarily-positive results were obtained at State public healthcare institution "Center on HIV prevention and infective diseases control" (Saint Petersburg) using enzyme-linked immunosorbent assay, consequently confirmed positive results of immunoblotting (IB). For order and control of carried out treatment, patients were examined by means of quantitative RNA HIV-1 determination using PCR method in real time mode.

The work was performed with application of the listed below test-systems with observance of the following manufacture protocol:

- ARCHITECT HIV Ag/Ab Combo (Abbott, USA), Chemiluminescence Immuno Assay;

- NEW LAV BLOT I (Bio-Rad, USA), lyzate immune blot (IB);

- HIV-1 p24-antigen-ELISA-BEST (Vector-Best, Russia), enzyme -linked immunosorbent assay;

- Abbott RealTime HIV-1 (Abbott, USA), PCR in real time mode.

Experimental investigations were carried out at specially equipped test bench, included a set of blood serum test samples in plastic tubes with biological media volume 1.0-1.8 ml, ultrasonic laboratory installation I100-840 (Russia), means of protection from aerosol cloud formation and disinfection. Samples of blood serum were prepared ahead of investigation performance; all the samples were in tubes Eppendorf (manufacturer Aptaka S.P. A. (Italy), material - polypropylene, volume $1.5 \mathrm{ml}$, diameter $10.5 \mathrm{~mm}$, height $40 \mathrm{~mm}$. For conducting ultrasonic investigation, laboratory installation was used in the range of $20-50 \mathrm{kHz}$ with different modes of exposure power (10-100\%) and time parameters of exposure from 30 to 300 seconds.

\section{Results and their discussion}

Methodically investigation was directed to study extinction levels of 28 positive by specific antigens to HIV presence in blood serum before and after ultrasonic exposure. Cut off level, obtained in the investigation, was used as criterion of positivity. Variations, associated with extinction growth of positive by specific antigens to HIV presence in blood serum, were registered in 12 cases. Results of this investigation, presented in tables 1 and 2, were obtained in the following conditions of ultrasonic exposure: characteristic of ultrasonic exposure relative power was in the range from 50 to $100 \%$, exposure time con- 
stituted 180-300 seconds, frequency - 22-44 kHz. Obtained in experimental ultrasonic exposure results are presented in table 1.

As it follows from the data presented in table 1, the largest absolute increase of extinction was registered for blood serum numbered from 9 to 12 , where the result of antibodies level presence exceeding upper threshold of test-system sensitivity (over 6.0 units of extinction) was obtained. Besides these results, considerable increase of blood serum extinction after ultrasonic exposure in samples with number $2(1.208)$ and $6(1,255)$ was registered. Extinction values in the rest cases have relatively small increase after ultrasonic exposure (USE) (from 0.010 to 0.179 ).

Table 1. Characteristic of extinction levels, positive by specific antigens to HIV presence in blood serum before and after ultrasonic exposure (USE)

\begin{tabular}{|c|c|c|c|c|c|}
\hline Item & $\begin{array}{c}\text { Extinction level } \\
\text { before USE } \\
\text { (OD/cut off) }\end{array}$ & $\begin{array}{c}\text { Time of USE } \\
\text { (sec.) }\end{array}$ & $\begin{array}{c}\text { Installation } \\
\text { power }(\%)\end{array}$ & $\begin{array}{c}\text { Frequency range } \\
\text { (kHz) }\end{array}$ & $\begin{array}{c}\text { Extinction level } \\
\text { before USE } \\
\text { (OD/cut off) }\end{array}$ \\
\hline 1 & 2 & 3 & 4 & 5 & 6 \\
\hline 1 & $4.765 / 0.254$ & $180-300$ & 100 & $22-44$ & $4.944 / 0.254$ \\
\hline 2 & $4.749 / 0.254$ & $180-300$ & 50 & $22-44$ & $5.957 / 0.254$ \\
\hline 3 & $4.862 / 0.254$ & $180-300$ & 50 & $22-44$ & $4.971 / 0.254$ \\
\hline 4 & $4.642 / 0.237$ & $180-300$ & 50 & $22-44$ & $4.652 / 0.237$ \\
\hline 5 & $5.044 / 0.237$ & $180-300$ & 50 & $22-44$ & $5.083 / 0.237$ \\
\hline 6 & $4.685 / 0.237$ & $180-300$ & 50 & $22-44$ & $5.940 / 0.237$ \\
\hline 7 & $0.5596 / 0.218$ & $180-300$ & 50 & $22-44$ & $0.5781 / 0.218$ \\
\hline 8 & $0.4285 / 0.218$ & $180-300$ & 50 & $22-44$ & $0.4437 / 0.218$ \\
\hline 9 & $4.895 / 0.226$ & $180-300$ & 50 & $22-44$ & $>6 / 0.226$ \\
\hline 10 & $4.876 / 0.226$ & $180-300$ & 50 & $22-44$ & $>6 / 0.226$ \\
\hline 11 & $4.528 / 0.226$ & $180-300$ & 50 & $22-44$ & $>6 / 0.226$ \\
\hline 12 & $4.833 / 0.226$ & $180-300$ & 50 & $22-44$ & $>6 / 0.226$ \\
\hline
\end{tabular}

The results of the investigation for the cases, when upper level of sensitivity was not surpassed (8 from 12 samples), were analyzed in respect to absolute increase of extinction level, as well as to relative (\%) variations, thereat extinction levels before exposure and cut off levels were compared (table 2).

Importance of the data, obtained in comparative analysis, is stipulated by surpassing of positivity threshold, demonstrating increase of extinction levels with regard to cut off. Maximal relative changes constituted 475.59 and $529.53 \%$, that is in $4-5$ times exceeds the level, necessary to recognize that blood serum as positive in respect of specific antibodies HIV antigens. Even more considerable results were obtained for blood serums, which overcame upper sensitivity threshold of tst-system (over 6.0 extinction units), tahat characterize 
Table 2. Characteristic of absolute and relative changes of extinction value, positive by specific antigens to HIV presence in blood serum before and after ultrasonic exposure

\begin{tabular}{|c|c|c|c|c|}
\hline Item & $\begin{array}{c}\text { Extinction level } \\
\text { before USE } \\
\text { (OD/cut off) }\end{array}$ & $\begin{array}{c}\text { Extinction level } \\
\text { before USE } \\
\text { (OD/cut off) }\end{array}$ & $\begin{array}{c}\text { Increase } \\
\text { of extinction after } \\
\text { USE (abs.) }\end{array}$ & $\begin{array}{c}\text { Increase of extinction } \\
\text { after USE } \\
\text { (total/cut off) }\end{array}$ \\
\hline 1 & $4.765 / 0.254$ & $4.944 / 0.254$ & 0.179 & $3.75 / 70.47$ \\
\hline 2 & $4.749 / 0.254$ & $5.957 / 0.254$ & 1.208 & $25.43 / 475.59$ \\
\hline 3 & $4.862 / 0.254$ & $4.971 / 0.254$ & 0.109 & $2.24 / 42.91$ \\
\hline 4 & $4.642 / 0.237$ & $4.652 / 0.237$ & 0.010 & $0.25 / 4.22$ \\
\hline 5 & $5.044 / 0.237$ & $5.083 / 0.237$ & 0.039 & $0.76 / 16.45$ \\
\hline 6 & $4.685 / 0.237$ & $5.940 / 0.237$ & 1.255 & $26.79 / 529.53$ \\
\hline 7 & $0.5596 / 0.218$ & $0.5781 / 0.218$ & 0.185 & $3.31 / 84.86$ \\
\hline 8 & $0.4285 / 0.218$ & $0.4437 / 0.218$ & 0.152 & $3.54 / 69.72$ \\
\hline
\end{tabular}

ultrasoni exposure as effective mean, capable to considerably change laboratory diagnostics results of HIV-infection.

\section{Conclusion}

The results obtained in experimental pilot investigation confirms possibility to obtain effect from disintegration of immune complexes with specific antibodies and antigens to HIV with capability of their further detection uning laboratory diagnostics methods immunofluorescent assay and immune-enzyme assay. That opportunity is mostly necessary for laboratory diagnostics of HIV-infection in acute period, in case of free antibodies deficiency in circulation.

\section{References}

1. Belyakov N. A. Immunodeficiency virus - danger to humans. Selected lectures of the University. St. Petersburg, 2020, vol. 206. 32 p. (In Russian)

2. Vzorov A.N. Immunobiology of HIV and abilities of the immune response against HIV-1, Moscow, MAKS Press, 2020. 87 p. (In Russian)

3. Lisitsina Z.N., Dmitriyevaskaya K. A., Koroban N. V., Kondrashova T. V. Immunological tests and diagnostics of acute HIV-infection. HIV-infection and Immunosuppressive Disorders, 2017, vol. 9, no. 2, pp. 36-41. (In Russian)

4. Saprygin D. B., Ivanov A.M. Laboratory diagnostics of infectious diseases. Reference. Moscow, 2016. 648 p. (In Russian)

5. Finogeev Yu. P., Lobzin Yu. V., Vinakmen Yu. A., Zakharenko S.M., Gromyko Yu. N., Uskov A.N. Clinical-laboratory diagnostics of infectious diseases. A guide for doctors. St. Petersburg, 2001. 384 p. (In Russian)

6. Bartlett Dzh., Gallant Dzh., Fam P. Clinical aspects of HIV-infection. Moscow, P. Valent Publ., 2012. 528 p.

7. Korolevskaya L. B., Shmagel K. V., Shmagel N. G. Characterization of circulating immune complexes in HIV-infected patients with different levels of viral load. Bulletin of Experimental Biology and Medicine, 2015, vol. 159, no. 4, pp. 460-463. (In Russian) 
8. Rjazanova G. A., Kocksin V.P., Khamzina R. V. "Free" and "bound" antibodies against HIV-1 structure proteins at early stage of the disease. Medical Immunology, 2005, vol. 7, no. 1, pp. 73-76. (In Russian)

9. Mitin Yu. A., Pastushenkov V.L., Drygin A.N. Ultrasonic action on biological objects of blood serum as a perspective direction in clinical laboratory diagnostics. Russian Biomedical Research, 2019, vol. 4, no. 2, pp. 18-21. (In Russian)

10. Mazus A.I., Kaminskiy G.D., Zimina V.N., Bessarab T.P., Pronin A.Yu., Tsyganova Ye. V., Ol'shanskiy A. Ya., Golokhvastova Ye. L., Tsarenko S. P., Shimonova T. Ye., Petrosyan T. R., Khalilulin T. R., Orlova-Morozova Ye. A., Serebryakov Ye. M., Nabiullina D. R., Ivanova T. V. National clinical recommendations for the diagnosis and treatment of HIV-infection in adults. Moscow, 2014. 75 p. (In Russian)

Authors'information:

Vladimir L.Pastushenkov — MD, Dr. Sci., Professor; pastprof@mail.ru Yuri A. Mitin - MD, Dr. Sci., Professor

Alexander V.Semenov - Dr. Sci.; alexvsemenov@yahoo.com

Diana E.Valutite - MD; dianavalutite008@gmail.com

Sergey Yu. Dudnikov - PhD

Madina M. Gabaeva - MD, PhD 